EPJ Web of Conferences 28, 12033 (2012)

DOI: $10.1051 /$ epjconf/20122812033

(C) Owned by the authors, published by EDP Sciences, 2012

\title{
Speculative Reading, Speculative Physics - the Ontology of the Large Hadron Collider
}

\author{
Clarissa A.L. Lee ${ }^{1}$ \\ ${ }^{1}$ Program in Literature, Duke University, 101 Friedl Building, Durham, NC 27701, USA
}

\begin{abstract}
The objective is to illustrate the hermeneutics of reading against the highly image-dependent (rather than object-oriented) world of experimental particle physics. Reading becomes the medium for mediating between the real and the virtual when constructing physical knowledge. "Virtual" particles, produced through material inter-course between machine and nature, are productive for rethinking the virtual/real entanglement. Reading enables a process of deconstructing and re-naturalizing microphysical trails, objects, and movements 'transformed' into machine code, post-detector. The process of reading is therefore the 'detection,' 'observation,' 'measurement,' 'abduction,' and 'interpretation' of coded information and knowledge; deciphered through data 'selected' for analysis, extrapolated as evidence for the confirmation/re-orientation of theoretical predictions, and included for the re-envisioning and revising of foundational explanations regarding the structure of modern physics. Reading as is explored here is about how reading as an act is inextricably linked with understanding one's perspective and the ideology one is immersed in, thus influencing how discovery is made and articulated through theory-construction.
\end{abstract}

\section{Reading Physics}

Reading is as much an act of cognition as it is of affective response between the physicist and the selection of subatomic world-trails he/she tracks. This affective connection is mediated as much by methodological choice as by the dominant paradigms that facilitate the reading that leads to the final interpretation. Reading is not an anthropomorphic prerogative but can also be performed by machines. Studying the different ways of reading, whether by machine or the human, provide us with insight into the different forms of reading involved in knowledge production, especially reading as an engagement with the abstract and highly symbolic narrative of the universe. It is hope that in the process of understanding how intrinsic and embedded reading is in epistemic-construction, and therefore, in understanding the ontology of the real and physical, that the act and method of reading can be advanced as a medium/tool for rigorous interdisciplinary exploration.

\section{Ontological Reading}

This primary stage of reading represents deep level reading that breaks down the signs of the text being read into its most rudimentary blocks. In physics, this may mean reading into the tacit knowledge, 'senseexperience' and affectivity forming the reasoning behind various epistemological commitments such as wavecorpuscularity, unification of forces, theory of relativity, counterfactual definiteness, unitarity, pointer states, decoherence, structure of the Standard Model, and symmetrical representations, among other interventions into the physical state.
Figure 1. Reading Feynman's (A)symmetry
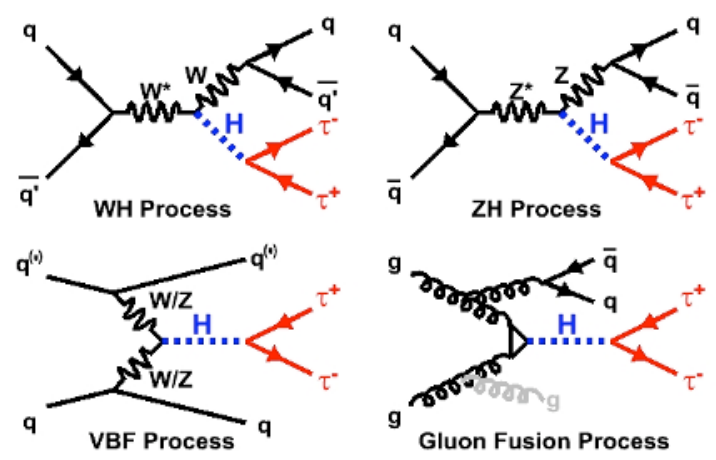

Ontological reading can also include the not-yetproperly-critiqued 'intuitive' or sense-directed reading. What is constructed as 'intuitive' form of knowledge is probably the most fundamental aspect of ontology while also the hardest to discern because of its self-reflexivity (due to a combination of abductive reasoning and 'irrational' insight that are only retroactively rationalized).

\section{1. 'Superficial’ versus ‘Deep’ Reading}

Ontological reading encompasses surface-layer (epidermic-level) reading and also deep-layer reading. When reading at the surface level, one first ascertains the signifying epistemics involved, while also marking and defining the boundary of semiotics embodying particular concepts, physical states, or specific macro representations of micro-physics. Deep-layer reading means discerning and teasing out the microscopic phenomena that are manifested at the macro-level but belong not to the macrostate. The Feynman diagrams above are representative of the deep reading of complex integration functions representing characteristics of the 
sub-atoms, even as these arcane equations are boundary markers defining the sub-structures of the sub-worlds of these particles.

\subsection{The Objective and the Subjective}

Ontological reading takes place at the level of the objective and the subjective. The objective here represents real observables that are replicable and accessible from more than one pathway, but whose probabilities are added to become a whole. Subjective reading, especially the reading of physically entangled states and Bayesian networks, enables access to the ontic. Ontic-level interpretation in physics looks into incomplete theories and narratives forming most physical structures whereby one can discern points of rupture, misapprehension and phenomenal paradoxes (that represent mis-recognition and boot-strapping of epistemic formations). An example of this is the history behind the construction of the Standard Model.

Fig. 2 Reading the continuum of the semi-classical
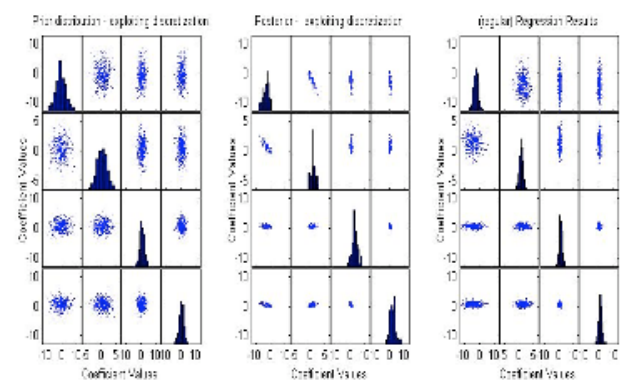

The figure above is an example of Bayesian Inference using Poisson Statistics - the process of inference forming is tied to ontological reading.

\section{Epistemological Reading}

By deriving and calculating the range of possibilities (through probabilities), the physicists are inscribing the path that can be taken by the 'narrative' provided by linepaths and integration of vertex points, which are also the inscription of particulate decay. The question remains as to whether mathematical 'diagramming,' or modelmaking, is an act of defamiliarization (acting as forms of distantiation through the suspension of the familiar), to augment one's access to the ontic, differentiate auxiliary events from primary narratives within the phenomenological processes, and create a paradigm of realism that is not based on anthropomorphic structures. It is at this juncture that the notion of the 'real' becomes problematic due to the different possibilities that the term offers. Moreover, one's position concerning the 'real' leads to the acceptance or rejection of outcomes, based on what is 'feasibly' replicable within the 'objective' world of empirical evidence.

Comparative reading, a form of epistemological reading, takes place when one reads between different sub-fields, either to transfer knowledge acquired within one's own subfield to another, or vice versa. It is in the process of comparative reading that one is able to uncover a crisis or knowledge gap surrounding existing paradigms since this involves the reading of experimental data or even simulated data against predictions and other existing models that are either popularly, or not, accepted.

\subsection{Interpreting/Mediating}

Epistemological reading involves the interpretation of information that has already passed through various mediating instruments, whereby a 'de-naturing' process occurs when data is broken down into different sections for tracking at different points and segments (measurements of electromagnetic energy and deposited hadronic energy deposited, track momenta, muon tracksegments; also, there are measurements in ionizing gas and quarks constituting certain hadrons). The separate parts that constitute information contained within a single data packet is read by separate sub-detectors that perform separate analyses with each 'strand' of the datum, later put together to illustrate developments in physical apprehension and ontological agreements.

Fig. 3 Machine-Epistemics

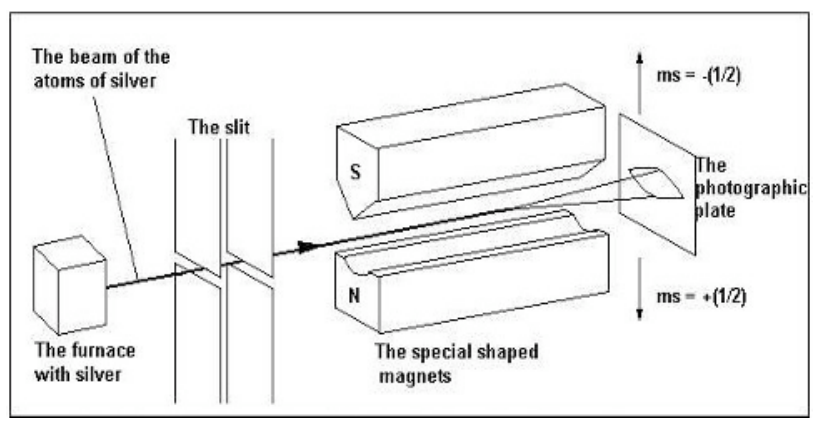

Stern-Gerlach experiment through inhomogenous magnetic field. Tracks can be seen on the photographic plates.

\subsection{Indeterminate Ideology}

Epistemological reading is the reading of uncertain and indeterminate information, as one cannot know in advance the exact route followed by the informational path beyond what is before us. However, as with any fiction that one reads, there is always an expectation that the indeterminacy can add-up to a determinate outcome (of 1).

Nevertheless, indeterminacy prevails even when one's cognitive praxis is taken into account. However, that boundary of possibilities is then narrowed to the confines and plausibility of one's scientific ideology, whether it is as simple as the choice of a theory or mathematical method. Hence, the level of indeterminacy in that outcome is influenced by choices made.

\subsection{Data and Cuts}

Fig. 4 Ontological Database 


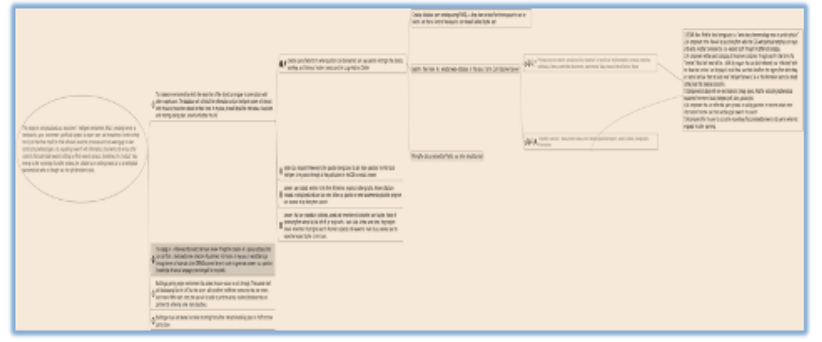

The diagram above represents a mind map, using the CDSWEB as an object, for building a database that can interact reflexively with the end-user, who is a researcher looking for ways to visualize and create a comprehensive map of different existing epistemic commitments and situate causality. A readable version can be found at http://www.duke.edu/ cal33 in the 'Researcher Tool' container.

Epistemological reading entails reading information obtained from events that have made through the final cut, mainly because there are many more events than what could be tracked. By reading the data against accepted frameworks, are we therefore narrowing the range of possibilities for new discoveries?

While the publications of any discovery build upon the work of previous experiments, research and interpretations, the ideology that connects these works are often buried in affirmative reports that mainly state certain assumptions made about any set of variables; so, one will have to follow that trail of cited publications in hope of finding the cause that initially sets the research program. However, when a publication attempts to dispute another one, that cause is more evidentially foregrounded.

\section{Entangling the ontological with the epistemological}

\subsection{Mathematical-Symbolic Reading}

\subsubsection{Interpreting Mathematical Operators}

The interpretation of the mathematics behind the physics structure is an example of ontological reading that is permeated with epistemological engineering. In order to understand the relationship between the different microphysical objects and elements in connection to space and time, especially when dealing with their manipulations of a space-time that is outside one's intuitive boundary, tensors and Dirac equations are used. They operate at the intersection of geometrical and algebraic visualization of interacting particles in ndimensional space by acting as the 'markers' for reading the 'action-map' of a universe constituting the signifying acts of scalars, vectors, spinors, and operators embodied in matrices, wave propagators and line functions. The equations utilized (from Schrödinger to Dirac to KleinGordon) demonstrate the possibility of more than one potential outcome, whereby some of these outcomes may be less than 'realistically' possible, in the sense that the outcome may be operating wholly within a 'virtual' realm that cannot be 'actualized.'
While most of the operators are attempts at symbolizing the potentiality and probable range of a physical reality, the complexity of certain physical states render it necessary for perturbations to be added to these states for proximal representation rather than depicting every micro detail to the smallest degree. While not representing the 'actual,' the perturbed models represent one's rationalization through one's mental model.

Fig. 5 Embodying Equations
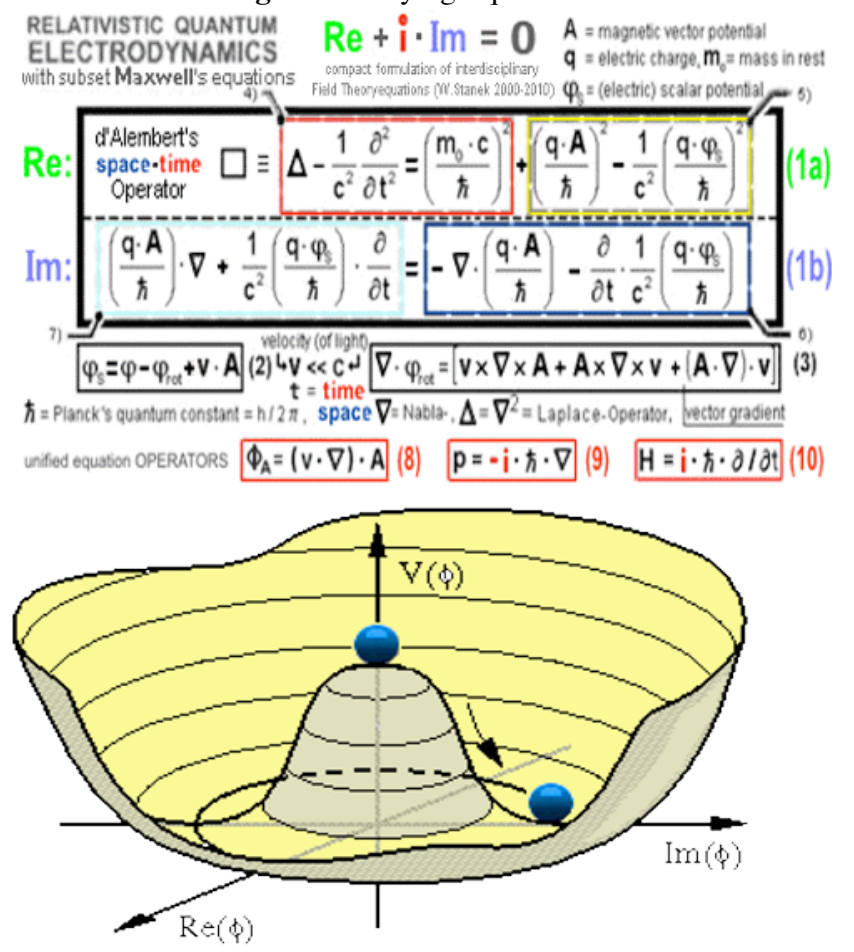

Importance of operators and matrices (above) as symbolic informants and signifying marks for expert readers navigating quantum field theory, and the 3-D graph (below) as visual representation of an otherwise complex equations of multidimension quality.

\subsubsection{Mathematical Model Construction}

By deriving and calculating the range of possibilities (through probabilities), the physicists are inscribing the path that can be taken by the 'narrative' provided by linepaths and integration of vertex points, which are also the inscription of particulate decay. The question remains as to whether mathematical 'diagramming,' or modelmaking, is an act of defamiliarization (acting as forms of distantiation through the suspension of the familiar), to augment one's access to the ontic, differentiate auxiliary events from primary narratives within the phenomenological processes, and create a paradigm of realism that is not based on anthropomorphic structures. It is at this juncture that the notion of the 'real' becomes problematic due to the different possibilities that the term offers. Moreover, one's position concerning the real leads to the acceptance or rejection of outcomes, based on what is 'feasibly' replicable within the 'objective' world of empirical evidence. The choice of privileging one set of mathematical apparatuses over another is also determined 
by the mathematics' ability to provide a 'reasonable' framework for illustrating data patterns or filling in narrative gaps in the theory. This is determined by the cognitive deixis of the expert reader and the shared cognitive map of the said reader's colleagues for being able to arrive at similar conclusions based on their own reading and subsequent analytic attempts. The modeling and simulation of thought experiments enable the sort of high-level speculation by moving beyond the need for corpo-realism. However, in building these fictional tools that also harness the power of mathematical narrative, the limits of explorations broaden.

\subsubsection{Macro versus the Micro}

Therefore, how does then one argue for interactions describing micro-phenomena that are 'hidden' and 'covered' over by their more visible macro counterparts, and how do we trust the veracity of knowledge presented only because it seemingly produces the desired results? One can argue that there is a relationship between mathematical inscription, expert readers of mathematical symbolism, and the mathematics of mechanical reproduction in a bid to provide 'machine' logic to the data before subjecting them to sophisticated mathematically-mediated theoretical testing in a bid to reconstruct the theories of the universe.

\subsection{Machine Reading}

\subsubsection{Instruments Writing History}

In the introduction to The Uses of Experiment, Gooding, Pinch and Schaffer pointed out how the effects of nature are rendered visible (or "realized") through "active instrument work" rather than merely passively observed in nature (4). Hence, the selection of the instrument is as much dictated by theory-choice as by accepted 'standards' that dictate the calibration of the instrument.

One may argue therefore that one can observe what has been calibrated to obtain through theoreticalpredictions; however, one may also return with results that are unexpected, or results that fail to obtain any measurable effects at all. But then, what if one wants to obtain that which lies outside the range of calibrated expectations (outside the $95 \%$ confidence level)? Can we be certain that the theory of choice is flexible enough to accommodate possibilities that may fall outside the constructed model?

Fig. 6 Inscribing Instruments

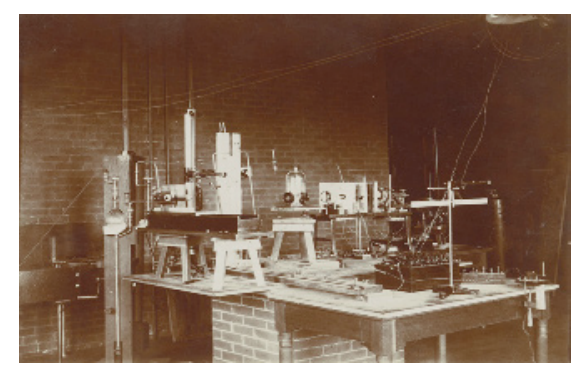

Volumes have been written on machine inscription and instrumentally produced inscriptions that are evidential traces of data. However, we have not found a useful way to observe how a machine, or that assemblage of machines called the Large Hadron Collider, performs that act of reading. We cannot follow every microscopic detail of the trail of injections and collisions to comprehend absolutely what goes on, which is a process of direct machine-reading, because to do so, would be equivalent to the situation of quantum wave collapse that prevents us from observing causality. Therefore, can one consider the process of machine reading as a process of effectual production?

Fig. 7 Scientific Heritage: Flight of the Bubble Chamber.

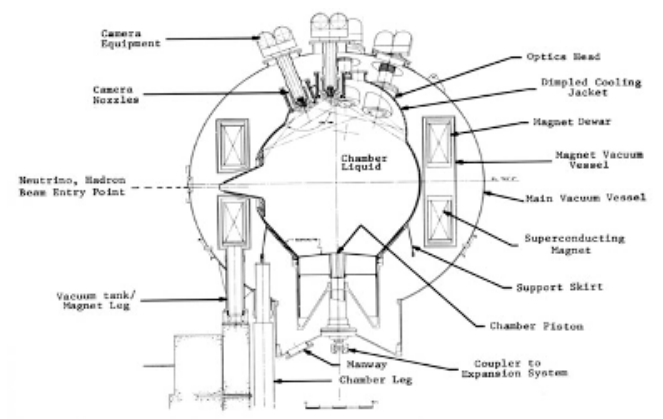

Instruments are designed and modeled out of specific assumptions regarding theories, the technological resources available, and the probable range of outcomes that the instrument is constructed to detect.

\subsubsection{Detecting/Reading}

Even if we find a way to observe the entire reading process, can we refrain from influencing the outcome of the reading, specifically since our understanding of reading is mediated by our perception of what that machine does; or is self-awareness sufficient? What if we are able to translate, into human language, all the raw data the detectors are able to collect and collate; data that are gibberish to us prior to instrumental translation and mediation, even to a well-trained eye. Can we presume that the machine language is merely an abstracted version of human language, or are there points where translations cannot take place, bringing about informational 'holes'? When we read the various monitors that tells us what the machine is seeing or feeling, we only read what has been processed. What about all the unprocessed material? What can be read at the very point of material contact between machine and nature, and what does the machine do to make sense of that can be crucial to helping us deal with ontological reading.

\section{References}

1. D. Gooding, T. Pinch, S Schaffer (eds.), The Uses of Experiments (Cambridge University Press, Cambridge, 1989).

2. R. Mann, An Introduction to Particle Physics and the Standard Model (CRC Press, Boca Raton, 2010).

3. M. Schlossahauer (ed.), Elegance and Enigma: The Quantum Interviews (Springer, Berlin-Heidelberg 2011). 